4

\title{
TEORÍA SOCIAL, NARRATIVA HISTÓRICA Y DISCIPLINA CIENTÍFICA
}

Aquiles Kobialka

DENOMINACIÓN DEL PROYECTO:

< APROXIMACIÓN A LOS ORÍGENES DISCIPLINARES DE LA HISTORIA DE LA CIENCIA EN LA UNIVERSIDAD NACIONAL DEL LITORAL (1930-1943) >

"Por sorprendente que nos pueda parecer, es posible edificar templos, palacios, incluso catedrales; excavar canales y construir puentes, desarrollar la metalurgia y la cerámica sin poseer un conocimiento científico o no teniendo sino sus rudimentos"

Alexandre Koyr 


\section{CUESTIONES INTRODUCTORIAS}

"La Historia de la Ciencia es una disciplina que, desde su ingreso al país, ha vivido en estado de crisis perpetua. Cultivada por pocos, con casi nula inserción en los programas de enseñanza universitaria, sin reconocimiento por parte del mundo académico, sobrevive dificultosamente, confundida por el público con la divulgación científica de corte histórico. Es que, a pesar de muchas asociaciones dedicadas al tema, la historia de la ciencia no ha acabado de profesionalizarse en el país, y como disciplina académica, se mueve dentro de cierta marginalidad. Este panorama poco alentador no implica, sin embargo, que no hayan existido o existan un buen número de investigaciones serias sobre la Historia de la Ciencia en la Argentina" [De Asúa,1993:7].

La extensa cita anterior nos sirve de síntesis acerca del estado de la cuestión. Así, y en esa línea, este trabajo fue pensado para contribuir a generar una identidad propia a la historia de la ciencia dentro del ámbito del historiador profesional, teniendo en cuenta que "teorías y descubrimientos en ciencias sociales son susceptibles de consecuencias prácticas (y políticas) más allá de que el [investigador] decida que se la pueda aplicar a determinada cuestión práctica" [Giddens, 1995:32].

Al respecto debemos decir que los relatos históricos testimonian que los países que influyeron en ámbitos de la cultura argentina, relacionados con la enseñanza de las ciencias en las primeras décadas del siglo XIX, eran aquéllos que en esos momentos lideraban el sistema científico tecnológico y que poseían una larga tradición de sistemas de gobierno liberal democráticos. Nos referimos a Inglaterra, Francia y cada vez con mayor preponderancia los Estados Unidos, países que desarrollaron institutos de investigación científica y, relacionado con ellos, una tradición de estudios sobre Historia de la Ciencia.

Hacia 1930 surgieron en Latinoamérica expectativas para un desarrollo acelerado de las ciencias, como parte de los proyectos de industrialización por sustitución de importaciones. La Argentina ocupaba, juntamente con Brasil, una posición de liderazgo en la región referido a desarrollo tecnológico e industrial. Consecuentemente, se intentó aquí constituir ámbitos de investiga- 
ción y enseñanza de historia y filosofía de la ciencia. Primero al incluir en el plan de estudios de la Facultad de Química Industrial y Agrícola, desde 1930, el cursado disciplinar bajo el nombre de Metodología e Historia de las Ciencias, materia que dependía del Instituto de Investigaciones Científicas y Tecnológicas de la citada Facultad. Finalmente, cuando logra su máximo status académico en 1939, como Instituto de Historia y Filosofía de la Ciencia, dependiente del Rectorado de la Universidad nacional del Litoral (U.N.L.), y que estuvo en actividad hasta 1943.

Presuponemos, de la lectura de los textos producidos en la época que se estudia, que quienes organizaron la institucionalización de la Historia de la Ciencia en la U.N.L. tenían el pensamiento que quien hace investigación científica no podía desconocer la historia de la ciencia. Entonces el proyecto del que formaba parte la Historia de la Ciencia estaba ligado a una formación universal. También pensamos que las relaciones entre la ciencia y la sociedad no pueden ser comprendidas sin desarrollar un entramado conceptual que dé cuenta de los conflictos que estas relaciones implican.

Quienes impulsaron esos proyectos(que se corporizaba, por ejemplo, en los departamentos universitarios y las sociedades profesionales y científicas), percibían esa disciplina como parte constitutiva de la infraestructura de la ciencia. Vale preguntarse hoy -para terminar esta introducción- a qué se debe la falta de profesionalización de la historia de la ciencia dentro del campo disciplinar de los historiadores.

\section{RESUMEN DEL PROYECTO}

Mediante este trabajo investigativo nos proponemos comprender el proceso -totalmente original en la Argentina y Sudamérica para su época- de estructuración e institucionalización de la Historia de la Ciencia como disciplina científica. También es propósito establecer la incidencia de los factores sociopolíticos en la formación del grupo de actores que instituyó la disciplina. 
Es por ello que decidimos fijarnos los siguientes objetivos específicos ${ }^{1}$ :

1. Elaborar el discurso histórico de forma lógico-sistemático, es decir basado en los elementos que componen el discurso histórico de las fuentes. Se expondrán hipótesis de trabajo y sus conclusiones sobre el modo en que la Historia de la Ciencia se instituye formando parte de un proyecto de Universidad, primero como Cátedra Universitaria en 1930 en la Facultad de Química Industrial y Agrícola (F.Q.I.y A.) de la U.N.L. y luego como Instituto de Investigación dependiente del Rectorado de esta Universidad en 1939, y por qué se interrumpe ese proyecto en 1943.

2. Analizar el proceso de conformación de la Historia de la Ciencia como campo disciplinar; sus elementos contextuales, la dinámica de los actores y los principales problemas que generó el mismo.

\section{SITUACIÓN MOTIVADORA}

Situamos nuestro trabajo de indagación histórica primeramente como un modo de discurso que se institucionalizó conformando una estructura de significación determinada que tiene que ser aprehendida en conexión con la dominación y la

\footnotetext{
${ }^{1}$ Con los siguientes Interrogantes que movilizan la investigación:

1) ¿Qué ideas científicas y filosóficas predominaban entre quienes concibieron la institucionalización de la disciplina Historia de la Ciencia?

2) ¿Puede afirmarse que los fundadores de la disciplina se agruparon por sus conocimientos y/o sus ideas políticas?

3) ¿Influyeron los cambios políticos nacionales e internacionales en la formación y cohesión del grupo? ¿Cómo afectaron esos cambios la estructura e institucionalización de los estudios de Historia de la Ciencia? ¿Qué grado de actualidad y exigencia se impuso a su estudio?

4) ¿Es posible establecer un perfil prosopográfico que describa y permita comprender la visión universitaria que tenía el grupo de profesores fundadores?

5) ¿Cuáles son las causas potencialmente sociales de la predilección en la creencia de la importancia de la Historia de la Ciencia, por las cuales la U.N.L. fue pionera en institucionalizarla en la Argentina? ¿Por qué ello se logró sólo parcialmente?

6) Si la institucionalización de la disciplina formaba parte de un proyecto ideológico y político, ¿fue suficiente la conducta social de los actores para que se consoliden como propiedades estructurales?
} 
legitimación. De allí deducimos que la institucionalización de la disciplina formaba parte de un proyecto ideológico y político que debe ser analizado.

El enfoque aplicado a la innovación que constituyó la Historia de la Ciencia como cátedra dependiente del Instituto de Investigaciones Científicas y Tecnológicas nos lleva a considerar la disciplina a instituir como una posibilidad entre otras, sujeta a controversias y negociaciones. En su estado naciente y polémico no se puede decidir aún sobre el objeto plenamente conformado. No obstante, es en esa etapa que deben tomarse las decisiones importantes (de investigación, estrategia política, estrategia educativa, etc.). Agregamos que los análisis sobre institucionalización del tipo aquí propuesto han sido especialmente cultivados en el último decenio en Francia y los Estados Unidos de Norteamérica por la influencia del auge de las ciencias sociales, que permiten alcanzar una mejor comprensión del fenómeno histórico en su conjunto.

En Argentina los estudios sociales de la ciencia están aún en sus comienzos.

\section{ANTECEDENTES SOBRE EL TEMA}

La temática que aborda este proyecto registra antecedentes tanto a nivel nacional (destacamos los trabajos de Carlos Galles, docente rosarino con actividades en el ámbito de la FIQ - UNL durante varios años, entre ellos: "la etapa santafesina del Archeion de Aldo Mieli") [De Asúa:1993] como internacional: en este ámbito, si bien abundan las investigaciones y publicaciones, nos pareció oportuno destacar a José M. López Piñero, investigador de la Universidad de Valencia, España, quien analiza la constitución de la historiografía de la ciencia, considerando los procesos de institucionalización disciplinar en varias universidades europea [Martínez (ed.):1994]. De cualquier manera, el tema específico: la institucionalización disciplinar como cátedra dependiente de la F.Q.I.y A. y a posteriori como Instituto de Historia y Filosofía de la Ciencia dependiente del Rectorado U.N.L. no ha sido abordado en forma conjunta hasta el presente. Concretamente, desconocemos estudios que si quiera hayan considerado la exposición de la implementación disciplinar en la mencionada F.Q.I.y A. 


\section{AVANCES EN LA FUNDAMENTACIÓN}

\subsection{Fundamentación Teórica}

El presente trabajo se enmarca en una historia social de las ciencias que es también una historia de los intentos realizados para modernizar, profesionalizar la enseñanza universitaria de las ciencias en Argentina, y para "modelar además en los alumnos un espíritu antidogmático, compensando en parte lo imperfecto de la inteligencia y evitando repetir penosamente los errores pasados mediante el estudio de la historia de la ciencia" [J.Gollán(h),1922:12].

A estos intentos institucionalizados los consideramos como un caso de conformación de un campo.

En esto recogemos el aporte de Pierre Bourdieu [2000:74], quien intenta romper con la falsa dicotomía de las perspectivas objetivistas (que se preocupan por rescatar estructuras, relaciones, leyes generales) y subjetivistas (pretenden rescatar el agente social sin tener en cuenta cómo incide el contexto en el que se desarrolla su práctica). Para este autor las dos perspectivas no son irreconciliables y propone el rescate de ambas a través de dos momentos para el análisis: relaciones objetivas y representaciones de los agentes.

En el primer momento intenta captar las relaciones objetivas (las estructuras de relaciones independientes de la conciencia y la voluntad de los individuos). Es un momento de objetivismo provisorio.

En el segundo momento rescata las representaciones que los agentes tienen de su propia práctica. Para dar cuenta de las luchas simbólicas que se dan en todos los campos, donde lo que está en juego es la producción de sentido, hay que tener en cuenta lo que dicen los agentes (su discurso). Lo diferente entre este segundo momento y el objetivismo es que los discursos son producidos por el agente social desde una posición en el campo. Es la verdad del agente social en relación con la posición que ocupa dentro del campo.

El hecho de ser un proceso de constitución o de construcción de un campo académico nos obliga a analizar cómo se estructura el sistema social en estudio.

\subsection{Hipótesis de trabajo}

La hipótesis de trabajo sustantiva que se procurará corroborar es la siguiente: 
"La institucionalización de la Historia de la Ciencia como disciplina científica durante el que hemos denominado periodo fundacional (1930-1943) en el ámbito de la U.N.L. no logró conformarse como una práctica social extendida en el tiempo, sino que fue un complejo proceso de asimilación de "la fuente luminosa original" constituida por la ciencia académica de los países capitalistas centrales, fenómeno que es el objeto de análisis de este trabajo de investigación y que en realidad, trasciende a la temática de la Historia de la Ciencia para transformarse en un problema más general de nuestra historiografía que recién en los últimos años ha comenzado a ser revisada sistemáticamente en esta dirección. 


\section{IMPACTO DE LA PROPUESTA}

Lo que destacamos como más importante, entre lo esperable tras finalizar este trabajo, se encuentra:

5.1. Contribuir a generar una identidad propia para la Historia de la Ciencia dentro del ámbito del historiador profesional.

5.2. Aportar al conocimiento de los procesos que establecieron tradiciones de investigación en el marco de la Universidad Nacional del Litoral.

5.3. Comprender el desarrollo de las sociedades actuales a través de la dilucidación histórica, social y conceptual de la ciencia.

Por último, es también aspiración de este trabajo llegar a ser una contribución a una comprensión más global de la historia de las ciencias y la cultura del país.

\section{ORIENTACIONES METODOLÓGICAS}

\section{Propuesta Metodológica}

No se pueden abordar las relaciones entre ciencia y sociedad y los conflictos implicados sin hacer uso de una metodología que permita exponer y poner de relieve precisamente estas cuestiones.

En ese orden de cosas, las actividades planificadas para alcanzar los objetivos específicos pueden resumirse en las siguientes:

6.1. Relevamiento y fichaje de las fuentes documentales.

6.2. Selección bibliográfica.

6.3. Realización de entrevistas orales.

6.4. Elaboración de guión-índice del trabajo.

6.5. Análisis del marco socio-histórico.

6.6. Relación de las fuentes con el marco socio-histórico.

6.7. Análisis prosopográfico.

6.8. Análisis formal o discursivo.

6.9. Desarrollo y redacción

A este estudio lo desarrollamos con ayuda de metodologías propiamente sociológicas, que permitan describir la lógica de constitución del campo científico, que es al mismo tiempo el proceso de constitución del campo de la Histo- 
ria de la Ciencia como disciplina escrita por los propios científicos.

A partir de ello, nosotros describiremos cómo las actividades en torno a la instauración de la disciplina adquieren forma por la "definición de la problemática" que tienen varios actores. Los actores principales, esto es, los que luchan por desarrollar su proyecto tratan de imponer sus definiciones de la problemática. El desarrollo de la innovación académica puede ser analizado en términos de lucha entre actores por imponer su definición particular del objeto a otros actores.

El seguimiento lo haremos sobre la base de analizar sus relaciones académicas y políticas, y ver cuales son los conceptos desde los que operaban. Destacamos las relaciones que estableció un actor, José Babini, quien en el periodo en estudio comienza a construir su posición en el campo de la historia de la ciencia - junto con la construcción del propio campo, logrando -según algunos autores- su máxima concreción intelectual con el establecimiento de su modelo disciplinar en los 50, cuando tras la muerte de Aldo Mieli - nombrado Director del Instituto de Historia y Filosofía de la Ciencia tras su creación en 1939 [Revista Universidad 5. Julio de 1939], toma la organización de la disciplina en el país. A pesar de ello, para ese entonces la Historia de la Ciencia ya había perdido el rol que la caracterizó en los 30: parte inescindible de la ciencia misma.

Por último, considerar como manifestamos la constitución del campo disciplinar de la historia de la ciencia como un espacio de lucha -y de cooperación también- implica la siguiente serie de análisis yuxtapuestos:

$>$ identificar a los agentes (o actores) en juego, quienes definen sus posiciones en un escenario específico;

$>$ interrogarse acerca del carácter específico del objeto historia de la ciencia.

$>$ definir el conjunto de elementos de tipo contextual (no en el sentido de ajenos a los alcances de los actores sino modificantes y modificables por estos) pertinentes para la visión global del proceso.

\section{Plan de actividades}

Las acciones 6.1 a 6.3 y 6.5 se desarrollarán durante el primer semestre de actividades; y las 6.4 y 6.6. a 6.9 durante el segundo. 
4.3. Referencias bibliográficas

\section{Fuentes Primarias}

$>$ Archivos

Biblioteca José Babini

Universidad Nacional Del Litoral

> Periódicos y revistas

Revista Universidad de la UNL

Revista de la Facultad de Química Industrial y Agrícola

Revista del Centro de Estudiantes de la F.Q.I. y A.

Revista Archeion de Historia de la Ciencia.

Publicación de Ley de creación de la U.N.L. 1919.

Publicaciones de la Cátedra de Historia de la Medicina de la U.B.A. 1937.

Publicación del Proyecto de reforma de los Estatutos Provisorios de la U.N.L. 1926.

Boletín de la F.Q.I. y A.

$>$ Entrevistas

Nicolás Babini.

Mario Benvenuto.

$>$ Documentos

Anales del Instituto de Investigaciones Científicas y Tecnológicas.

Anales de la Sociedad Científica de Santa Fe.

Anales de la Sociedad Científica Argentina.

Actas del Congreso Universitario Argentino 1936.

Actas Comisión Especial Reforma Plan de Estudios 1930. F.Q.I. y A. UNL. 
Actas Comisión Discusión del Proyecto de Estatuto Propio U.N.L 1935.

Orientaciones de la F.Q.I. y A. (por Josué Gollán [h]) 1922.

Artículos Inéditos obtenidos en Biblioteca de la Fundación José Babini

\section{Fuentes Secundarias}

$>$ Marco teórico

Martínez E. (ed.) Ciencia, Tecnología y Desarrollo: Interrelaciones Teóricas y Metodológicas, Nueva Sociedad, Caracas, 1994

La Fuente y Ortega H. (eds.) Mundialización de la Ciencia y Cultura Nacional, Universidad Autónoma de Madrid, Doce Calles, Madrid, 1993

REDES Revista de Estudios Sociales en Ciencias, U.N.Q. Quilmes, Diciembre 1994.

Bourdieu P. Cosas Dichas, Gedisa, Madrid, 1988.

Bourdieu P. Razones prácticas, Gedisa, Madrid, 1994.

Bourdieu P. Sociología y Cultura, Grijalbo, México, 1990.

Bourdieu P. El Oficio de Sociólogo. Gedisa, Madrid, 1994.

Bourdieu P. Los usos sociales de la ciencia, Nueva Visión, B.A., 2000

De Asúa M. La Ciencia en la Argentina. C.E.A.L, B.A. 1993.

Giddens A. La constitución de la sociedad, Amorrortu, B.A., 1995.

Moradielos, E. El Oficio de Historiador, SXXI, México, 1997.

Sabino C. Cómo se hace una tesis, Lumen, B.A., 1994.

$>$ General

Babini N., José Babini en Páginas para una autobiografía, A.B.J.B., B.A., 1992.

Babini J. Historia de la Ciencia en la Argentina, Solar, B.A., 1986.

González F. Las Caras del Leviathán, Anthropos, Barcelona, 1992.

La Política de Investigación Científica y Tecnológica Argentina. Historia y Perspectivas, CEAL, 1992.

"ILUIL" Boletín de la Sociedad Española de Historia de la Ciencia Vol. 7 № 13, Facultad de Ciencias Matemáticas, Zaragoza, 1984.

Saldaña J. (Coordinador) Historia Social de la Ciencia en América Latina, Porrúa Grupo Editor, México, 1996.

Oteiza E. Vessuri H. Estudios Sociales de la Ciencia y la Tecnología en América Latina, C.E.A.L, B.A., 1993.

Macor D. y Iglesias E. El peronismo antes del peronismo, U.N.L., S.F., 1997.

“Estudios Sociales” № 4. Revista universitaria semestral, U.N.L, S.F. 1993. 